\title{
STABILIZATION OF LINEAR SYSTEMS BY TIME-DELAY FEEDBACK CONTROLS*
}

\author{
BY \\ JIONGMIN YONG \\ Purdue University
}

\begin{abstract}
The notion of $r$-stabilizability of linear autonomous systems is introduced. If the system $[A, B]$ is completely controllable and the spectrum of $A$ is contained in the left half of the complex plane then the system is $r$-stabilizable for any $r>0$. Finally, a one-dimensional case is discussed.
\end{abstract}

1. Introduction. We consider the following system

$$
\dot{x}(t)=A x(t)+B u(t),
$$

where $A$ is an $(n \times n)$-matrix, $B$ is an $(n \times m)$-matrix, $x \in \mathbf{R}^{n}, u \in \mathbf{R}^{m}$, and $t \in \mathbf{R}$. (Throughout this paper, all the matrices and vectors are real.) It is well known that (see [11], for example) if $[A, B]$ is completely controllable, then for any set $\Lambda=$ $\left\{\lambda_{1}, \lambda_{2}, \ldots, \lambda_{n}\right\} \subset \mathbf{C}$, with the property that

$$
\Lambda^{*} \stackrel{\Delta}{=}\left\{\bar{\lambda}_{1}, \bar{\lambda}_{2}, \ldots, \bar{\lambda}_{n}\right\}=\Lambda,
$$

there exists an $(m \times n)$-matrix $K$ such that

$$
\sigma(A+B K)=\Lambda \text {. }
$$

Here, $\sigma(A+B K)$ denotes the spectrum of $A+B K$. In particular, if we let $\Lambda \subset \mathbf{C}^{-} \stackrel{\Delta}{=}\{\lambda$ $\in \mathbf{C} \mid \operatorname{Re} \lambda<0\}$, then, under the following feedback control,

$$
u(t)=K x(t),
$$

the system (1.1) is asymptotically stable.

A feedback control of the form (1.4) is not quite realistic because we usually have time delays. Thus, instead of (1.4) we should consider a feedback control of the form

$$
u(t)^{\prime}=K x(t-r),
$$

*Received June 23, 1986.

This research was supported by the David Ross Fellowship of Purdue University. 
where $r>0$ is a time lag. By substituting (1.5) into (1.1) we get

$$
\dot{x}(t)=A x(t)+B K x(t-r) .
$$

By [10], we know that, if we choose $K$ such that the system

$$
\dot{x}(t)=(A+B K) x(t)
$$

is asymptotically stable, then for sufficiently small $r>0,(1.6)$ is also asymptotically stable. On the other hand, in [5] it was proved that if the system

$$
\dot{x}(t)=A x(t)+A_{0} x(t-r)
$$

is asymptotically stable for all $r>0$, then

$$
\sigma(A) \subseteq \mathbf{C}^{-} .
$$

Thus, if (1.9) is not satisfied, or equivalently, if the system

$$
\dot{x}(t)=A x(t)
$$

is not asymptotically stable, then for any given $K$, the system (1.6) can not be asymptotically stable for all $r>0$. Hence, a natural question can be asked. For any given $r>0$, does there exist a $K$ such that (1.6) is asymptotically stable? This is the question we will study in this paper.

2. Preliminaries. For any entire function $H(z)$, we let

$$
\mathscr{N}(H) \equiv \mathscr{N}(H(\cdot)) \stackrel{\Delta}{=}\{z \in \mathbf{C} \mid H(z)=0\} .
$$

We denote a system of form (1.8) by $\left[A, A_{0}, r\right]$. We define

$$
p\left(\lambda ; A, A_{0} ; r\right)=\operatorname{det}\left(\lambda I-A-A_{0} e^{-r \lambda}\right) .
$$

Definition 2.1. Let $r>0$ and $[A, B]$ be given. System $[A, B]$ is said to be $r$-stabilizable if there exists a $K$ such that $[A, B K ; r]$ is asymptotically stable.

It is well known that (see [3]) system $\left[A, A_{0} ; r\right]$ is asymptotically stable if and only if

$$
\mathscr{N}\left(p\left(\cdot ; A, A_{0} ; r\right)\right) \subseteq \mathbf{C}^{-} .
$$

Let us observe the following:

$$
\begin{aligned}
p\left(\lambda ; A, A_{0} ; r\right) & \equiv \operatorname{det}\left(\lambda I-A-A_{0} e^{-r \lambda}\right) \\
& =\frac{1}{r^{n}} \operatorname{det}\left((r \lambda) I-r A-r A_{0} e^{-(r \lambda)}\right) \\
& =\frac{1}{r^{n}} p\left(r \lambda ; r A, r A_{0} ; 1\right) .
\end{aligned}
$$

Thus, (2.3) is equivalent to the following:

$$
\mathscr{N}\left(p\left(\cdot, r A, r_{\star} \mathbf{i}_{1} ; 1\right)\right) \subseteq \mathbf{C}^{-} .
$$

Hence, we get

Proposition 2.2. Let $r>0,[A, B]$ be given. Then, $[A, B]$ is $r$-stabilizable if and only if $[r A, B]$ is 1-stabilizable. 
This proposition shows that we only need to consider the case that $r=1$. Now, for $r=1$, we have

$$
\begin{aligned}
p\left(\lambda ; A, A_{0} ; 1\right) & =P(\lambda)+Q(\lambda) e^{-\lambda} \\
& =e^{-\lambda}\left[e^{\lambda} P(\lambda)+Q(\lambda)\right] \equiv e^{-\lambda} H(\lambda),
\end{aligned}
$$

where $\operatorname{deg} P=n, \operatorname{deg} Q \leqslant n-1$, and $P(\lambda)$ has the form

$$
P(\lambda)=\lambda^{n}+\cdots \text {. }
$$

It is clear that

$$
\mathscr{N}(H)=\mathscr{N}\left(p\left(\cdot ; A, A_{0} ; 1\right)\right) .
$$

Definition 2.3. An entire function $H(\lambda)$ is said to be stable if

$$
\mathscr{N}(H) \subseteq \mathbf{C}^{-} \text {. }
$$

Thus, from (2.8), we know that system $\left[A, A_{0} ; 1\right]$ is asymptotically stable if and only if $H(\lambda)$ defined by $(2.6)$ is stable.

We now quote some basic results, which we will use in the sequel, about the stability of some elementary transcendental functions.

Let $h(z, t)$ be a polynomial of the form

$$
h(z, t)=P(z) t+Q(z),
$$

where $P(z), Q(z)$ are polynomials, $\operatorname{deg} P=n, \operatorname{deg} Q \leqslant n-1$, and $P$ has the form (2.7). Let

$$
H(z)=h\left(z, e^{z}\right) \text {. }
$$

Then, for $z=i y, y \in \mathbf{R}$, we have real-valued functions $F(y)$ and $G(y)$, such that

$$
H(i y)=F(y)+i G(y) .
$$

Then, we have the following theorem due to Pontryagin [9]. (See also [1] and [4].)

THEOREM 2.4. If $H(z)$ is stable, then all the zeros of $F(y)$ and $G(y)$ are real and alternating. Moreover,

$$
G^{\prime}(y) F(y)-G(y) F^{\prime}(y)>0
$$

for all $y \in \mathbf{R}$. Conversely, $H(z)$ is stable if one of the following holds.

(a) All the zeros of $F(y)$ and $G(y)$ are real and alternating and (2.13) holds for at least one point $y$.

(b) All the zeros of $F(y)$ are real, and for each zero $y=y_{0}$,

$$
F^{\prime}\left(y_{0}\right) G\left(y_{0}\right)<0 \text {. }
$$

(c) All the zeros of $G(y)$ are real, and for each zero $y=y_{0}$,

$$
G^{\prime}\left(y_{0}\right) F\left(y_{0}\right)>0 \text {. }
$$

To close this section, we make the following simple remark.

REMARK 2.5. Since $H(z)$ is entire and takes real values on the real-axis, by the Schwarz Reflection Principle, we know that $F(y)$ is an even function and $G(y)$ is an odd function. 
3. Fundamental lemmas. We are going to prove two fundamental results in this section. These results will play a crucial role in proving our main result.

In this section, we always assume that $H(z)$ is of the form (2.11) and that $F(y)$ and $G(y)$ are defined by (2.12).

Lemma 3.1. Suppose $H(z)$ is stable. Then, there exists a $c \in \mathbf{R}$ such that

$$
H_{1}(z) \stackrel{\Delta}{=} z H(z)+c
$$

is stable.

Proof. Since $H(z)$ is stable, by Remark 2.5, we have

$$
0 \neq H(0)=F(0)+i G(0)=F(0) .
$$

Thus, without loss of generality, we assume $F(0)>0$. By (3.1), we have

$$
\begin{aligned}
H_{1}(i y) & =i y(F(y)+i G(y))+c \\
& =[-y G(y)+c]+i y F(y) \\
& =F_{1}(y)+i G_{1}(y),
\end{aligned}
$$

where

$$
\left\{\begin{array}{l}
F_{1}(y)=c-y G(y) \\
G_{1}(y)=y F(y) .
\end{array}\right.
$$

Since $H(z)$ is stable, by Theorem 2.4 , we know that all the zeros of $F(y)$ are real. Thus, all the zeros of $G_{1}(y)$ are real.

Now, we choose $c \in \mathbf{R}$ such that

$$
0<c<\frac{1}{2} \inf \left\{\left|y_{0} G\left(y_{0}\right)\right| \mid F\left(y_{0}\right)=0\right\} .
$$

Note that the right-hand side of (3.5) is positive because it is easy to see that

$$
\inf _{y \in \mathbf{R}}|H(i y)|>0 .
$$

By choosing such a $c$, we have

$$
G_{1}^{\prime}(0) F_{1}(0)=F(0) c>0 .
$$

Also, for any $y_{0} \in \mathscr{N}(F)$, we have

$$
\begin{aligned}
G_{1}^{\prime}\left(y_{0}\right) F_{1}\left(y_{0}\right) & =\left(F\left(y_{0}\right)+y_{0} F^{\prime}\left(y_{0}\right)\right)\left(c-G\left(y_{0}\right) y_{0}\right) \\
& =y_{0} F^{\prime}\left(y_{0}\right)\left(c-y_{0} G\left(y_{0}\right)\right) \\
& \geqslant-y_{0}^{2} F^{\prime}\left(y_{0}\right) G\left(y_{0}\right)-\left|c y_{0} F^{\prime}\left(y_{0}\right)\right| \\
& \geqslant-y_{0}^{2} F^{\prime}\left(y_{0}\right) G\left(y_{0}\right)-\frac{1}{2}\left|y_{0} G\left(y_{0}\right) \cdot y_{0} F^{\prime}\left(y_{0}\right)\right| \\
& =-\frac{1}{2} y_{0}^{2} F^{\prime}\left(y_{0}\right) G\left(y_{0}\right)>0 .
\end{aligned}
$$

Here, we have used (2.13). Since it is clear that

$$
\mathscr{N}\left(G_{1}\right)=\mathscr{N}(F) \cup\{0\},
$$

it follows from (c) of Theorem 2.4 that $H_{1}(z)$ is stable. 
From the proof, we see that $c$ is explicitly determined by the following:

$$
\left\{\begin{array}{l}
F(0) c>0, \\
|c|<\inf \left\{\left|y_{0} G\left(y_{0}\right)\right| \mid y_{0} \in \mathscr{N}(F)\right\} .
\end{array}\right.
$$

Lemma 3.2. Suppose $H(z)$ is stable. Then, for any $a>0$, there exist $b, c \in \mathbf{R}$, such that

$$
H_{2}(z)=\left(z^{2}+a^{2}\right) H(z)+b z+c
$$

is stable.

Proof. From (3.11), we have

$$
\begin{aligned}
H_{2}(i y) & =\left(a^{2}-y^{2}\right) H(i y)+b i y+c \\
& =\left(a^{2}-y^{2}\right)(F(y)+i G(y))+b y i+c \\
& =\left[\left(a^{2}-y^{2}\right) F(y)+c\right]+i\left[\left(a^{2}-y^{2}\right) G(y)+b y\right] \\
& =F_{2}(y)+i G_{2}(y),
\end{aligned}
$$

where

$$
\left\{\begin{array}{l}
F_{2}(z)=\left(a^{2}-y^{2}\right) F(y)+c \\
G_{2}(z)=\left(a^{2}-y^{2}\right) G(y)+b y .
\end{array}\right.
$$

Now, we have, by the stability of $H(z)$, that

$$
0 \neq H(i a)=F(a)+i G(a) .
$$

Thus, we have two cases.

Case I. $F(a) \neq 0$. Then, we take

$$
\left\{\begin{array}{l}
c=0 \\
b F(a)>0 \\
|b| \leqslant \frac{1}{2} \inf \left\{\frac{\left|a^{2}-y_{0}^{2}\right|}{\left|y_{0}\right|}\left|G\left(y_{0}\right)\right| \mid y_{0} \in \mathscr{N}(F)\right\} \stackrel{\Delta}{=} \delta_{1} .
\end{array}\right.
$$

By an argument similar to that used in the proof of Lemma 3.1, we have $\delta_{1}>0$. It is clear that

$$
\mathscr{N}\left(F_{2}\right)=\mathscr{N}(F) \cup\{ \pm a\} .
$$

Thus, all zeros of $F_{2}$ are real. Observe that

$$
F_{2}^{\prime}(y) G_{2}(y)=\left\{\left(a^{2}-y^{2}\right) F^{\prime}(y)-2 y F(y)\right\}\left[\left(a^{2}-y^{2}\right) G(y)+b y\right] .
$$

Hence, we have, by (3.15), that

$$
F_{2}^{\prime}(a) G_{2}(a)=-2 a F(a) \cdot b a=-2 a^{2} b F(a)<0 .
$$

By Remark 2.5, $F(y)$ is an even function. Thus,

$$
F_{2}^{\prime}(-a) G_{2}(-a)=-2 a^{2} b F(-a)=-2 a^{2} b F(a)<0 .
$$


Now, for any $y_{0} \in \mathscr{N}(F)$, we have

$$
\begin{aligned}
F_{2}^{\prime}\left(y_{0}\right) G_{2}\left(y_{0}\right) & =\left(a^{2}-y_{0}^{2}\right) F^{\prime}\left(y_{0}\right)\left[\left(a^{2}-y_{0}^{2}\right) G\left(y_{0}\right)+b y_{0}\right] \\
& \leqslant-\left(a^{2}-y_{0}^{2}\right)^{2}\left|F^{\prime}\left(y_{0}\right) G\left(y_{0}\right)\right|+\left|b y_{0}\right|\left|F^{\prime}\left(y_{0}\right)\right|\left|\left(a^{2}-y_{0}^{2}\right)\right| \\
& \leqslant-\frac{1}{2}\left(a^{2}-y_{0}^{2}\right)^{2}\left|F^{\prime}\left(y_{0}\right) G\left(y_{0}\right)\right|<0 .
\end{aligned}
$$

Here, we have used (2.13). Thus, by Theorem 2.4, part (b), we get the stability of $\mathrm{H}_{2}(z)$.

Case II. $G(a) \neq 0$. Then, we take

$$
\left\{\begin{array}{l}
b=0, \\
c G(a)<0, \\
|c| \leqslant \frac{1}{2} \inf \left\{\left|a^{2}-y_{0}^{2}\right|\left|F\left(y_{0}\right)\right| \mid y_{0} \in \mathscr{N}(G)\right\} \stackrel{\Delta}{=} \delta_{2} .
\end{array}\right.
$$

An argument similar to that used above gives $\delta_{2}>0$. In this case, we have

$$
\mathscr{N}\left(G_{2}\right)=\mathscr{N}(G) \cup\{ \pm a\}
$$

We have

$$
F_{2}(a) G_{2}^{\prime}(a)=c(-2 a G(a))=-2 a c G(a)>0 .
$$

Since $G(y)$ is an odd function (by Remark 2.5), we also have

$$
F_{2}(-a) G_{2}^{\prime}(-a)=2 a c G(-a)=-2 a c G(a)>0 .
$$

Now, for any $y_{0} \in \mathscr{N}(G)$, by (3.21) and (2.15), we have

$$
\begin{aligned}
F_{2}\left(y_{0}\right) G_{2}^{\prime}\left(y_{0}\right) & =\left[\left(a^{2}-y_{0}^{2}\right) F\left(y_{0}\right)+c\right]\left(a^{2}-y_{0}^{2}\right) G^{\prime}\left(y_{0}\right) \\
& \geqslant\left(a^{2}-y_{0}^{2}\right)^{2} F\left(y_{0}\right) G^{\prime}\left(y_{0}\right)-\left|c\left(a^{2}-y_{0}^{2}\right) G^{\prime}\left(y_{0}\right)\right| \\
& \geqslant \frac{1}{2}\left(a^{2}-y_{0}^{2}\right)^{2} F\left(y_{0}\right) G^{\prime}\left(y_{0}\right)>0 .
\end{aligned}
$$

Thus, by Theorem 2.4, part (c), $H_{2}(z)$ is stable.

\section{Stabilization.}

4.1. Single-input case. Suppose we are given a single-input system $[A, b]$ which is completely controllable. Then, it is well known that (cf. [11]) we have the following canonical representation:

$$
A=\left(\begin{array}{ccccc}
0 & 1 & & & \\
& 0 & \ddots & & \\
& & \ddots & \ddots & 1 \\
-a_{0} & -a_{1} & \ldots & -a_{n-2} & -a_{n-1}
\end{array}\right), \quad b=\left(\begin{array}{c}
0 \\
0 \\
\vdots \\
0 \\
1
\end{array}\right)
$$

THEOREM 4.1. Suppose $[A, b]$ is completely controllable, and

$$
\boldsymbol{\sigma}(A) \subseteq \mathbf{C}^{-} \cup \mathbf{C}^{0} \equiv\{\lambda \in \mathbf{C} \mid \operatorname{Re} \lambda \leqslant 0\} .
$$

Then, for any $r>0,[A, b]$ is $r$-stabilizable. 
Proof. We first prove that $[A, b]$ is 1 -stabilizable. We let

$$
k=\left(-k_{0},-k_{1}, \ldots,-k_{n-1}\right)^{T} \in \mathbf{R}^{n} .
$$

Then we have, by assuming (4.1), that

$$
\begin{aligned}
p\left(\lambda ; A, b k^{T} ; 1\right) & \equiv \operatorname{det}\left(\lambda I-A-b k^{T} e^{-\lambda}\right) \\
& =\lambda^{n}+a_{n-1} \lambda^{n-1}+\cdots+a_{0}+e^{-\lambda}\left(k_{n-1} \lambda^{n-1}+\cdots+k_{0}\right) \\
& =e^{-\lambda}\left\{e^{\lambda}\left(\lambda^{n}+a_{n-1} \lambda^{n-1}+\cdots+a_{0}\right)+\left(k_{n-1} \lambda^{n-1}+\cdots+k_{0}\right)\right\} .
\end{aligned}
$$

By (4.2), we can assume that

$$
\lambda^{n}+a_{n-1} \lambda^{n-1}+\cdots+a_{0}=\lambda^{\prime} P_{1}(\lambda) P_{2}(\lambda),
$$

where $l \geqslant 0$ and

$$
\left\{\begin{array}{l}
\mathscr{N}\left(P_{1}\right) \subseteq \mathbf{C}^{-} \equiv\{\lambda \in \mathbf{C} \mid \operatorname{Re} \lambda<0\} \\
\mathscr{N}\left(P_{2}\right) \subseteq \mathbf{C}^{0} \backslash\{0\} \equiv\{\lambda \in \mathbf{C} \backslash\{0\} \mid \operatorname{Re} \lambda=0\}
\end{array}\right.
$$

We claim that there exists a polynomial, $Q_{1}$, with real coefficients and $\operatorname{deg} Q_{1}<l$, such that

$$
H(\lambda) \stackrel{\Delta}{=} \lambda^{\prime} e^{\lambda}+Q_{1}(\lambda)
$$

is stable. If $l=0$, we take $Q_{1}(\lambda) \equiv 0$. If $l>0$, consider $e^{\lambda}$ which is of the form (2.11) and is stable. By Lemma 3.1, there exists a $c_{1} \in \mathbf{R}$, such that $\lambda e^{\lambda}+c_{1}$ is stable. Again there exists a $c_{2} \in \mathbf{R}$ such that

$$
\lambda\left(\lambda e^{\lambda}+c_{1}\right)+c_{2}=\lambda^{2} e^{\lambda}+c_{1} \lambda+c_{2}
$$

is stable. By induction, our claim follows. Now, by applying Lemma 3.2, and using a similar argument as above, we can find a polynomial $Q_{2}(\lambda)$ with real coefficients and with $\operatorname{deg} Q_{2}<\operatorname{deg} P_{2}$, such that $P_{2}(\lambda) H(\lambda)+Q_{2}(\lambda)$ is stable. The polynomial $Q$ defined by

$$
Q(\lambda)=Q_{1}(\lambda) P_{2}(\lambda)+Q_{2}(\lambda)
$$

then has real coefficients, and satisfies

$$
\begin{aligned}
\operatorname{deg} Q & \leqslant \max \left\{\operatorname{deg} Q_{1}+\operatorname{deg} P_{2}, \operatorname{deg} Q_{2}\right\} \\
& =\operatorname{deg} Q_{1}+\operatorname{deg} P_{2}<l+\operatorname{deg} P_{2} .
\end{aligned}
$$

If we let the vector $k^{T}=\left(k_{0}, \ldots, k_{n-1}\right)$ in $R^{n}$ be defined by

$$
k_{n-1} \lambda^{n-1}+\cdots+k_{0}=P_{1}(\lambda) Q(\lambda) \text {, }
$$

we have

$$
\begin{aligned}
p\left(\lambda ; A, b k^{T} ; 1\right) & =e^{-\lambda}\left\{e^{\lambda} \lambda^{\prime} P_{1}(\lambda) P_{2}(\lambda)+P_{1}(\lambda) Q(\lambda)\right\} \\
& =e^{-\lambda} P_{1}(\lambda)\left\{e^{\lambda} \lambda^{\prime} P_{2}(\lambda)+Q_{1}(\lambda) P_{2}(\lambda)+Q_{2}(\lambda)\right\} \\
& =e^{-\lambda} P_{1}(\lambda)\left\{\left[\lambda^{\prime} e^{\lambda}+Q_{1}(\lambda)\right] P_{2}(\lambda)+Q_{2}(\lambda)\right\} \\
& =e^{-\lambda} P_{1}(\lambda)\left\{P_{2}(\lambda) H(\lambda)+Q_{2}(\lambda)\right\}
\end{aligned}
$$


which is stable. Thus, we have proved that $[A, b]$ is 1-stabilizable. Now, for any $r>0$, we know that $[r A, b]$ is still completely controllable and also we have

$$
\sigma(r A) \subseteq \mathbf{C}^{-} \cup \mathbf{C}^{0} .
$$

Thus, by the above proof, we know that $[r A, b]$ is 1-stabilizable. Thus, by Proposition 2.2, $[A, b]$ is $r$-stabilizable.

4.2. Multi-input case. Now, we consider a multi-input system $[A, B]$ which is completely controllable. Then, by p. 44 of [11], we have the following representation.

$$
A=\left(\begin{array}{cccc}
A_{1} & & & \\
& A_{2} & & \\
& & \ddots & \\
& & & A_{l}
\end{array}\right)_{n \times n}, \quad B=\left(\begin{array}{ccccc}
b_{11} & b_{12} & \cdots & b_{1 l} & * \\
& b_{22} & \cdots & b_{2 l} & * \\
& & \ddots & \vdots & \vdots \\
& & & b_{l l} & *
\end{array}\right)_{n \times m,}
$$

where, for $1 \leqslant j \leqslant l$,

$$
A_{j}=\left(\begin{array}{ccccc}
0 & 1 & & & \\
& 0 & \ddots & & \\
& & & 0 & 1 \\
-a_{0}^{j} & -a_{1}^{j} & \cdots & -a_{n,-2}^{j} & -a_{n_{j}-1}^{j}
\end{array}\right)_{n, \times n_{j}} \quad b_{j j}=\left(\begin{array}{c}
0 \\
0 \\
\vdots \\
0 \\
1
\end{array}\right)_{n_{j} \times 1},
$$

and $*$ represents the entries in which we are not interested. It is clear that in this case, we must have $m \geqslant l$. Now, we let

$$
K=\left(\begin{array}{c}
\hat{K} \\
0
\end{array}\right)_{m \times n,}
$$

where

$$
\hat{K}=\left(\begin{array}{llll}
k_{11}^{T} & & & \\
& k_{22}^{T} & & \\
& & \ddots & \\
& & & k_{l l}^{T}
\end{array}\right)_{l \times n} k_{j j} \in \mathbf{R}^{n_{j}}, 1 \leqslant j \leqslant l .
$$

Then, we have

$$
A+B K=\left(\begin{array}{cccc}
A_{1} & & & \\
& A_{2} & & \\
& & \ddots & \\
& & & A_{l}
\end{array}\right)+\left(\begin{array}{cccc}
b_{11} k_{11}^{T} & b_{12} k_{22}^{T} & \cdots & b_{1 l} k_{l l}^{T} \\
& b_{22} k_{22}^{T} & \cdots & b_{2 l} k_{l l}^{T} \\
& & \ddots & \vdots \\
& & & b_{l l} k_{l l}^{T}
\end{array}\right) .
$$

Thus, we get

$$
p(\lambda ; A, B K ; r)=\prod_{j=1}^{l} p\left(\lambda ; A_{j}, b_{j j} k_{j j}^{T} ; r\right),
$$

and we have the following result. 
THEOREM 4.2. Suppose $[A, B]$ is completely controllable and

$$
\boldsymbol{\sigma}(A) \subseteq \mathbf{C}^{-} \cup \mathbf{C}^{0} .
$$

Then, for any $r>0,[A, B]$ is $r$-stabilizable.

Proof. By (4.15), we see that $\left[A_{j}, b_{j j}\right]$ is completely controllable for each $1 \leqslant j \leqslant l$. Also, we have that

$$
\sigma\left(A_{j}\right) \subseteq \sigma(A) \subseteq \mathbf{C}^{-} \cup \mathbf{C}^{0}, \quad 1 \leqslant j \leqslant l .
$$

Thus, by Theorem 4.1, for any $r>0$, we can find $k_{j j}$, such that $p\left(\lambda ; A_{j}, b_{j j} k_{j j}^{T} ; r\right)$ is stable for $1 \leqslant j \leqslant l$. Hence, by (4.19), we get the $r$-stabilizability of $[A, B]$.

5. One-dimensional case. In this section, we consider a one-dimensional system

$$
\dot{x}(t)=a x(t)+u(t),
$$

where $a \in \mathbf{R}$. By Proposition 2.2, we know that (5.1) is $r$-stabilizable for $r>0$, if and only if for some $k \in \mathbf{R}$

$$
\dot{x}(t)=\operatorname{rax}(t)+k x(t-1)
$$

is asymptotically stable. It is easy to see that

$$
\begin{aligned}
p(\lambda ; r a, k ; 1) & =\lambda-r a-e^{-\lambda} k \\
& =e^{-\lambda}\left[e^{\lambda}(\lambda-r a)-k\right] .
\end{aligned}
$$

Let us cite the following theorem due to Hayes.

THEOREM 5.1 ([6]). All the roots of

$$
z e^{z}-p e^{z}-q=0
$$

have negative real parts (where, $p, q \in \mathbf{R}$ ) iff

(a) $p<1$,

(b) $p<-q<\sqrt{a_{1}^{2}+p^{2}}$

where, for $p=0, a_{1}=\pi / 2$; and for $p \neq 0,0<a_{1}<\pi, a_{1}=p \tan a_{1}$.

As a consequence of Hayes' Theorem, we have

THEOREM 5.2. System (5.1) is $r$-stabilizable iff

$$
r a<1 .
$$

Proof. We know that system (5.1) is $r$-stabilizable if and only if for some $k \in \mathbf{R}$ (see (5.3)),

$$
e^{\lambda}(\lambda-r a)-k=0
$$

has no roots in $\mathbf{C}^{+} \cup \mathbf{C}^{0}$. Then, we take $p$ to be $r a$ and we can find $k \equiv q$ satisfying (b) of Theorem 5.1. Hence our theorem follows.

Moreover, for system (5.1), we have the following:

TheOREM 5.3. Suppose $k \in \mathbf{R}, r>0$, such that $[a, k ; r]$ is asymptotically stable. Then, for any $\hat{r} \in[0, r],[a, k ; \hat{r}]$ is also asymptotically stable. 
REMARK 5.4. This theorem says that if $k$ can be used to $r$-stabilize system (5.1), then it also can be used to $\hat{r}$-stabilize (5.1), for any $\hat{r} \in[0, r]$.

Proof of Theorem 5.3. In our case, by Theorem 5.1, we have

$$
\left\{\begin{array}{l}
r a<1 \\
r a<-r k<\sqrt{a_{1}^{2}+r^{2} a^{2}},
\end{array}\right.
$$

where, for $a=0, a_{1}=\pi / 2$; for $a \neq 0,0<a_{1}<\pi, a_{1}=r a \tan a_{1}$. Then, we have

$$
a+k<0 \text {. }
$$

This implies that $[a, k ; 0]$ is asymptotically stable. Now, we consider $\hat{r} \in(0, r]$. It is clear that (see (5.7))

$$
\left\{\begin{array}{l}
\hat{r} a<1 \\
\hat{r} a<-\hat{r} k
\end{array}\right.
$$

are always true. Thus, we only need to show that

$$
-\hat{r} k<\sqrt{\hat{a}_{1}^{2}+\hat{r}^{2} a^{2}},
$$

where, for $a=0, \hat{a}_{1}=\pi / 2$; for $a \neq 0,0<\hat{a}_{1}<\pi, \hat{a}_{1}=\hat{r} a \tan \hat{a}_{1}$.

Case 1. $a=0$.

Then, we have $a_{1}=\hat{a}_{1}=\pi / 2$, and thus (5.10) is equivalent to

$$
-r k<\frac{\pi}{2} \text {. }
$$

This is true because by (5.7) and $\hat{r} \in(0, r]$, we have

$$
-k<\frac{\pi}{2 r} \leqslant \frac{\pi}{2 \hat{r}} \text {. }
$$

Thus, our theorem is true for this case.

Case 2. $a \neq 0$.

In order to have (5.10), it suffices to have

$$
\sqrt{\left(\frac{a_{1}}{r}\right)^{2}+a^{2}} \leqslant \sqrt{\left(\frac{\hat{a}_{1}}{\hat{r}}\right)+a^{2}}
$$

i.e.,

$$
\frac{a_{1}^{2}}{r^{2}} \leqslant \frac{\hat{a}_{1}^{2}}{\hat{r}^{2}},
$$

or, equivalently, by the definitions of $a_{1}$ and $\hat{a}_{1}$,

$$
\tan ^{2} a_{1} \leqslant \tan ^{2} \hat{a}_{1} \text {. }
$$

(1) $a>0$. We consider the function $h(s)$ defined implicitly by

$$
h(s)=s \tan h(s), \quad 0<s<1,0<h(s)<\frac{\pi}{2} .
$$

Since

$$
\begin{aligned}
h^{\prime}(s) & =\tan h(s)+s \sec ^{2} h(s) \cdot h^{\prime}(s) \\
& =\tan h(s)+\frac{h(s)}{\tan h(s)} \sec ^{2} h(s) \cdot h^{\prime}(s) \\
& =\tan h(s)+\frac{2 h(s)}{\sin 2 h(s)} \cdot h^{\prime}(s),
\end{aligned}
$$


thus

$$
h^{\prime}(s)=\tan h(s) \cdot\left[1-\frac{2 h(s)}{\sin 2 h(s)}\right]^{-1}<0, \quad 0<s<1, \quad 0<h(s)<\frac{\pi}{2},
$$

i.e., $h(s)$ is decreasing. Thus

$$
0<a_{1}=h(r a) \leqslant h(\hat{r} a)=\hat{a}_{1}<\frac{\pi}{2} .
$$

Hence, (5.15) follows.

(2) $a<0$. We consider the function $h(s)$ defined implicitly by

$$
h(s)=s \tan h(s), \quad-\infty<s<0, \frac{\pi}{2}<h(s)<\pi .
$$

We have

$$
h^{\prime}(s)=\tan h(s) \cdot\left[1-\frac{2 h(s)}{\sin 2 h(s)}\right]^{-1}<0, \quad-\infty<s<0, \frac{\pi}{2}<h(s)<\pi,
$$

since $\tan h(s)<0, \sin 2 h(s)<0$ for $\pi / 2<h(s)<\pi$. Thus, $h(s)$ is decreasing. Noting $r a \leqslant \hat{r} a$, we have

$$
\pi>a_{1}=h(r a) \geqslant h(\hat{r} a)=\hat{a}_{1}>\frac{\pi}{2} .
$$

Since $\tan x$ is increasing and negative for $x \in(\pi / 2, \pi)$, we get (5.15). Hence our theorem is proved.

6. Some remarks. First of all, from the previous sections, we can easily prove the following result.

Theorem 6.1. Suppose $[A, B]$ is completely controllable, and

$$
\boldsymbol{\sigma}(A) \subseteq \mathbf{C}^{-} \cup \mathbf{C}^{0} \cup\{a\},
$$

where $a>0$, and the Jordan blocks of $A$ corresponding to $a$ are of order 1 . Then, for any $r>0$, with $r a<1$, the system $[A, B]$ is $r$-stabilizable.

Secondly, we should note that Theorem 5.3 is not true in general for the higher-dimensional case. To see this, we have the following.

Theorem 6.2. Let $a>0$. Then, there exists a $c \in \mathbf{R}$ such that

$$
e^{\lambda}\left(\lambda^{2}+a^{2}\right)+c
$$

is stable iff

$$
\sin a \neq 0 \text {. }
$$

The proof is just a modification of that of Lemma 3.2.

Now, we consider a two-dimensional system with

$$
A=\left(\begin{array}{cc}
0 & 1 \\
-a^{2} & 0
\end{array}\right), \quad b=\left(\begin{array}{l}
0 \\
1
\end{array}\right)
$$

and $\sin a \neq 0, a>\pi$. Then, we have $c \in \mathbf{R}$ such that (6.2) is stable. Thus, if we take

$$
k=\left(\begin{array}{c}
-c \\
0
\end{array}\right)
$$


then we have

$$
p\left(\lambda ; A, b k^{T} ; 1\right)=\left[e^{\lambda}\left(\lambda^{2}+a^{2}\right)+c\right] e^{-\lambda},
$$

which is stable. Thus, $\left[A, b k^{T} ; 1\right]$ is asymptotically stable. However, if we take

$$
0<r=\frac{\pi}{a}<1
$$

then $\sin (r a)=0$. Thus, by Theorem 6.2,

$$
\begin{aligned}
p\left(\lambda ; A, b k^{T} ; r\right) & =e^{-\lambda r}\left(\lambda^{2}+a^{2}\right)+c \\
& =\frac{e^{-\lambda r}}{r^{2}}\left\{e^{(\lambda r)}\left[(r \lambda)^{2}+(r a)^{2}\right]+r^{2} c\right\}
\end{aligned}
$$

can not be stable; i.e., $\left[A, b k^{T} ; r\right]$ is not asymptotically stable.

Finally, let us suggest some natural questions to be solved.

(1) From the discussion of the one-dimensional case (Theorem 5.2), it seems true that a completely controllable system $[A, B]$ is $r$-stabilizable iff

$$
r \cdot \sup \{\operatorname{Re} \lambda \mid \lambda \in \sigma(A)\}<1 .
$$

(2) Under what conditions on $A$ does Theorem 5.3 remain true for the system $[A, B]$ ?

Acknowledgment. The author would like to thank Professor L. D. Berkovitz for some help.

\section{REFERENCES}

[1] R. Bellman and K. L. Cooke, Differential-Difference Equations, Academic Press, New York, London, 1963

[2] R. Datko, A procedure for determination of the exponential stability of certain differential-difference equations, Quart. Appl. Math. 36, 279-292 (1978)

[3] L. E. El'sgol'ts and S. B. Norkin, Introduction to the theory and application of differential equations with deviating arguments, Academic Press, New York, London, 1973

[4] J. K. Hale, Functional Differential Equations, Springer-Verlag, New York, Berlin, 1977

[5] J. K. Hale, E. F. Infante, and F. P. Tsen, Stability in linear delay equations, J. Math. Anal. Appl. 105, 535-555 (1985)

[6] N. D. Hayes, Roots of the transcendental equation associated with a certain difference-differential equation, J. London Math. Soc. 25, 226-232 (1950)

[7] Li, Xun-jing, On the absolute stability of systems with time lags, Acta Math. Sinica 13, 558-573 (1963), English transl. in Chinese Math. 4, 609-626 (1963)

[8] J. M. Mahaffy, A test for stability of linear differential delay equations, Quart. Appl. Math. 40, 193-202 (1982)

[9] L. S. Pontryagin, On the zeros of some elementary transcendental functions, Amer. Math. Soc. Transl., Ser. 2, 1, 95-110 (1955)

[10] Yuan-Xun Qin, Iong-qing Liou, and Lian Wang, Effect of time-lags on stability of dynamical systems, Sci. Sinica 9, 719-747 (1960); English transl. in Chinese Math. 9, 169-198 (1967)

[11] W. M. Wonham, Linear multivariable control: A geometric approach, 2nd ed., Springer-Verlag, New York, Heidelberg, Berlin, 1979 